\title{
Analysis of co-extrusion process for preparation of gradient index plastic optical fiber
}

\author{
Jyh-Ping Hsu*, Sung-Hwa Lin \\ Department of Chemical Engineering, National Taiwan University, Taipei 10617, Taiwan, ROC
}

Received 30 May 2003; received in revised form 27 June 2003; accepted 27 June 2003

\begin{abstract}
The fabrication of gradient index plastic optical fiber in a closed co-extrusion process is simulated theoretically. The concentration dependency of the diffusivities of monomers in host polymer is taken into account on the basis of a modified free volume theory together with a generalized Flory-Huggins theory for a three-component polymer solution. The applicability of the model derived is justified by fitting it to the experimental data reported in the literature, and its performance is found to be satisfactory. We show that the diffusion of a mobile phase in a polymer solution is dependent upon the structure and the concentration of polymer, and the concentration of the other mobile phase present.
\end{abstract}

(C) 2003 Elsevier Ltd. All rights reserved.

Keywords: Gradient index optical fiber; Closed co-extrusion; Concentration-dependent diffusivity

\section{Introduction}

Investigations on the refractive properties of materials have been conducted for over thousand years since human beings are interested on astronomical refraction problems. To predict exactly the path of light in a refractive material, however, is impossible until Snell's law and Fermat's principle were proposed hundreds of years ago. The possibility of applying an inhomogeneous refractive medium in an optical system was not considered until Maxwell [1] proposed his spherical symmetric focus lens, the so-called Maxwell's fisheye lens. Maxwell's result was modified and generalized by Luneburg [2], Morgan [3], and numerous researchers decades later. In the past decades, various theoretical studies were performed on the utilization of distributed elements such as the imaging lenses for microscopes, telescopes, and photographic lenses, and the optical fiber for the transmitting of light [1].

Using a gradient index optical element has the advantages such as low image aberration and torsion, sharp imaging, small number of elements, and high adjustability. Although theoretical investigations on gradient index media

\footnotetext{
* Corresponding author. Tel.: + 886-223-637-448; fax: + 886-223-623040 .

E-mail address: jphsu@ccms.ntu.edu.tw (J.P. Hsu).
}

are ample in the literature, technology counterparts are still very limited. Techniques for fabricating high quality, low cost, and desired gradient index distribution materials are not developed fast enough to keep pace with theoretical predictions. In his experiment for the verification of the Schmidt's theory of sun, Wood [1] prepared the first gradient index element, a radial-distributed index lens of gelatin, which was prepared by diffusion of glycerin (higher refractive index) from the middle of gelatin. Until present, most of the reported results for distributed refractive index elements are based upon glass and polymer plastic fibers. Glass optical fiber can be produced in principle by exchanging ions with different refractive indices between glass and surrounding solution [4]. The fabrication of stressfree, discolored glass optical fiber used as wave guides was not successful, however, until 1970s. Techniques such as ion implantation, electron irradiation, vapor deposition, and others, have also been proposed for the preparation of colorless, low-loss glass wave guides [1]. Due to its high cost, low production rate, poor adjustability, and poor processibility, glass optical fiber tends to be replaced by plastic optical fiber. Although the optical loss of the latter was found to be significant in the early stage of its development, subsequent study showed that this drawback can be alleviated [5]. Various processes are reported to be efficient for the fabrication of plastic optical fiber including, 
for example, suspension copolymerization [6,7], internal diffusion and surface evaporation (IDSE) [8-11], and multilayer co-extrusion [12-15].

The performance of an optical fiber depends largely on its refractive index distribution. That of a cylindrical optical fiber with a parabolic refractive index distribution in the radial direction, for example, was found to be satisfactory in light imaging [1]. A parabolic type refractive index distribution, $n_{\mathrm{D}}$, can be described by

$n_{\mathrm{D}}=n_{\mathrm{D} 0}\left(1-A r^{* 2}\right)$,

where $n_{\mathrm{D} 0}$ is the highest refractive index, which occurs on the axis of the fiber, and $r^{*}$ and $A$ are the scaled radial distance and a constant, respectively. In practice, a refractive index distribution can be controlled in fabrication because it correlates with the composition of a fiber as [16]

$n_{\mathrm{D}}=\left(\frac{1+2 \Phi}{1-\Phi}\right)^{1 / 2}$

$\Phi=\frac{\sum_{i}\left[\left(n_{\mathrm{D} i}^{2}-1\right) /\left(n_{\mathrm{D} i}^{2}+2\right)\right]\left(w_{\mathrm{p} i} / d_{\mathrm{p} i}\right)}{\sum_{i}\left(w_{\mathrm{p} i} / d_{\mathrm{p} i}\right)}$

In these expressions, $n_{\mathrm{D} i}$ and $d_{\mathrm{p} i}$ are, respectively, the refractive index and the density of polymer component $i$, and $w_{\mathrm{p} i}$ is its mass fraction. The key step common to the processes for preparing plastic optical fiber is the diffusion of monomers in polymer gels. For simplicity, the diffusivities of monomers in a polymer solution are usually assumed as constant [17-19]. Although analytical results can be derived based on this assumption, it is known that the diffusivity of a monomer in a polymer solution depends not only on the concentration and the structure of polymer, but also on the concentrations of other monomers present [20, 21]. Because these effects can be significant in practice, an analysis, which takes them into account, is highly desirable. This is conducted in the present study.

\section{Analysis}

A schematic representation of the optical fiber fabrication process under consideration is illustrated in Fig. 1 [14, 15,17]. Poly-methyl methacrylate (PMMA) with monomers methyl methacrylate (MMA) and benzyl methacrylate (BzMA) are placed in reservoir tank 1, and PMMA with monomer MMA are placed in reservoir tank 2; these tanks are kept at $60{ }^{\circ} \mathrm{C}$. The polymer solution in tank 1 is extruded at a volumetric flow rate of $0.5 \mathrm{~cm}^{3} / \mathrm{min}$ by a gear pump into the inner layer of the concentric die, and that in tank 2 is pumped synchronously at a volumetric flow rate of $1.5 \mathrm{~cm}^{3} /$ min into the outer layer of the die. Diffusion of MMA and BzMA occurs when the polymer solutions pass through the
1

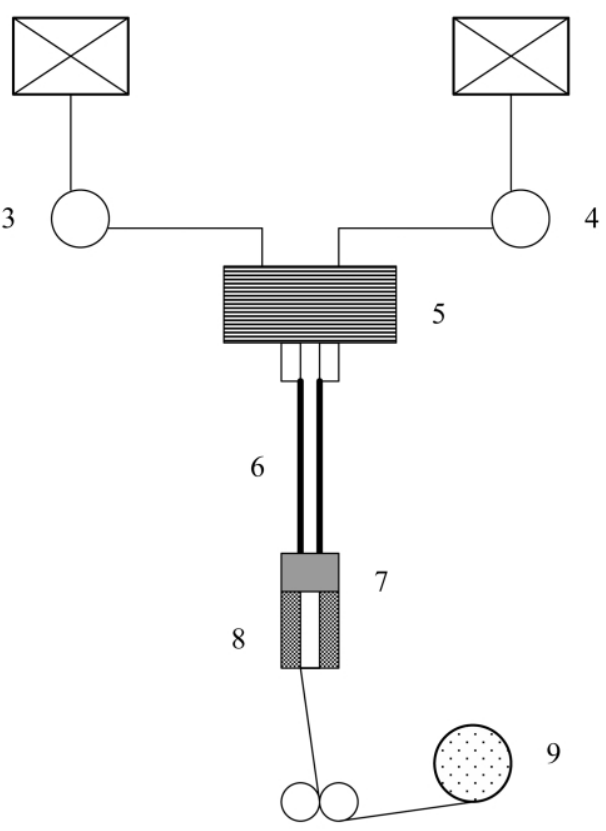

Fig. 1. Schematic representation of the experimental apparatus of the closed extrusion process considered. 1 and 2, material supply tanks, 3 and 4, gear pumps, 5, concentric die, 6, enclosed (diffusion) zone, 7, core-shell separator, 8 , hardening zone, 9 , take-up roll [14,15].

enclosed (diffusion) zone. After passing this zone, the polymer solutions are hardened by $220 \mathrm{~V}$ UV light to fix the refractive index distribution. A take-up roller then collects the finished product. The change in the bulk density of the filament is assumed to be negligible. Referring to Fig. 2, $R_{i}$ and $R_{\mathrm{o}}$ are, respectively, the radii of the inner and the outer layer of the diffusion zone, $r$ and $z$ are, respectively, the radial distance and the distance from the top of the diffusion zone, and $L$ is the total length of the diffusion zone in the $z$ direction. Under steady state operation, the mass transfer of monomer is described by

$$
\nabla \cdot\left(\rho_{i} u_{i}\right)=\nabla \cdot\left[\rho_{i}\left(u_{i}-u\right)+\rho_{i} u\right]=-\nabla \cdot\left(D_{l} \nabla \rho_{i}\right)+\nabla \cdot\left(\rho_{i} u\right)=0
$$

where subscript $i=1$ and 2 represent, respectively, monomers MMA and BzMA, $\rho_{i}$ and $w_{i}$ are, respectively, the mass concentration and the mass fraction of monomer $i$, and $u_{i}$ and $D_{i}$ are, respectively, the velocity and the diffusion coefficient of monomer $i$, and $u$ is the velocity of bulk solution. The system under consideration is azimuthally symmetric, and the only non-zero component of $u$ is its $z$ component, $u_{z}$, the constant exclusion speed. We assume that both the diffusion of polymer and that of monomers in the $z$-direction are negligible, and the variation of the density of polymer solution is insignificant. Therefore, Eq. (3) can be rewritten in cylindrical coordinates as 


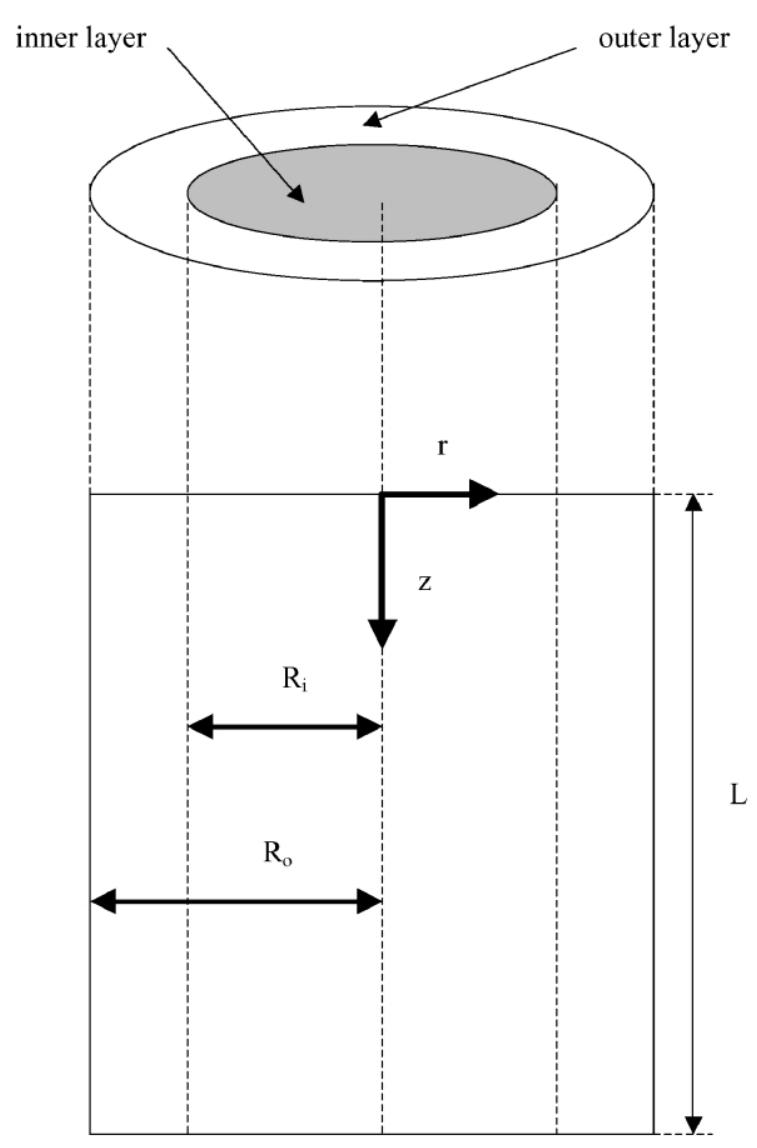

Fig. 2. Coordinates used in theoretical analysis.

$$
\begin{aligned}
u_{z} \frac{\partial w_{i}}{\partial z} & =\frac{1}{r} \frac{\partial}{\partial r}\left(r D_{i} \frac{\partial w_{i}}{\partial r}\right) \\
& =D_{i} \frac{\partial^{2} w_{i}}{\partial r^{2}}+\frac{\partial D_{i}}{\partial r} \frac{\partial w_{i}}{\partial r}+\frac{1}{r} D_{i} \frac{\partial w_{i}}{\partial r}, \quad i=1,2
\end{aligned}
$$

The boundary conditions associated with this equation are assumed as

$$
\frac{\partial w_{i}}{\partial r}=0, r=0 \quad \text { and } \quad r=R_{\mathrm{o}}, \quad i=1,2
$$

$w_{1}=w_{1 i}, \quad 0<r<R_{i}, \quad z=0$

$w_{1}=w_{1 o}, \quad R_{i}<r<R_{o}, z=0$

$w_{2}=w_{2 i}, 0<r<R_{i}, z=0$

$w_{2}=0 R_{i}<r<R_{\mathrm{o}}, \quad z=0$

In these expressions, $w_{1 i}$ and $w_{2 i}$ are, respectively, the mass fractions of MMA and BzMA in the inner layer at the top of the diffusion zone, and $w_{10}$ is the mass fraction of MMA in the outer layer at the top of the diffusion zone. Eq. (4a) implies that the diffusion of each component is azimuthally symmetric and the wall of the diffusion zone is impenetrable to both MMA and BzMA.

For the present three-component polymer solution, the free volume fraction, $f$, can be expressed as

$$
\begin{aligned}
f & =\phi_{1} f_{1}+\phi_{2} f_{2}+\left(1-\phi_{1}-\phi_{2}\right) f_{3} \\
& =\left(f_{1}-f_{3}\right) \phi_{1}+\left(f_{2}-f_{3}\right) \phi_{2}+f_{3} \\
& =\alpha_{f 1} \phi_{1}+\alpha_{f 2} \phi_{2}+f_{3}
\end{aligned}
$$

where $f_{i}$ is the free volume fraction of component $i$ at pure state, and $f_{3}$ is that of PMMA. In the equation above, the volume fraction of component $i, \phi_{i}$, can be expressed as

$\phi_{i}=\frac{w_{i} / d_{i}}{\frac{w_{1}}{d_{1}}+\frac{w_{2}}{d_{2}}+\frac{1-w_{1}-w_{2}}{d_{3}}}$

where $d_{i}$ is the density of component $i$. Based on Fujita et al.'s theory [22] for the thermodynamic diffusivity of monomer in a polymer solution, a free volume theory for transporting property, and an extension of Flory-Huggins equation for three-component polymer solution (Appendix A), the diffusivities of MMA and BzMA in PMMA, $D_{1}$ and $D_{2}$, can be expressed, respectively, as

$D_{1}=D_{0,1} \exp \left(-\frac{f_{\mathrm{c}}}{f}+\frac{f_{\mathrm{c}}}{f_{3}}\right)\left[1-\left(1-1 / z_{2}\right) \phi_{1}-2 \chi_{1} \phi_{2}\right]$

$D_{2}=D_{0,2} \exp \left(-\frac{f_{\mathrm{c}}}{f}+\frac{f_{\mathrm{c}}}{f_{3}}\right)\left[1-\left(1-z_{2}\right) \phi_{2}-2 \chi_{2} \phi_{2}\left(1-\phi_{2}\right)\right]$

Here, $D_{0, i}$ is the dilute diffusivity of monomer $i, f_{\mathrm{c}}$ is the critical free volume fraction for the co-operative movement of the polymer segments, $z_{i}=\left(m_{i} / d_{i}\right) /\left(m_{1} / d_{1}\right), m_{i}$ being the molecular weight of component $i$, and $\chi_{i}$ is the FloryHuggins parameter of component $i$. Because the variation of the concentration of polymer is limited, $f_{\mathrm{c}}$ is assumed to be constant. Eqs. (5), (7a), and (7b) simulate the effect of the presence of one monomer on the diffusivity of the other monomer. If the concentrations of MMA and BzMA are both low, the retardation effects arising from polymer chains are about the same for both monomers. In this case, the main factors that are significant to the transport property ratio of different monomers are the molecular weights and the activation energy of monomers (Appendix A). The latter is equivalent to the interaction energy between a monomer molecule and its neighboring molecules. The dilute 
diffusivity ratio of monomers, then, can be estimated by

$$
\begin{aligned}
\frac{D_{0,2}}{D_{0,1}}= & \left(\frac{m_{1}}{m_{2}}\right)^{1 / 2} \\
& \exp \left\{-\frac{U_{23}^{*}-U_{13}^{*}}{k T}\left[\left(1-f_{3}\right)^{4}-2\left(1-f_{3}\right)^{2}\right]\right\}
\end{aligned}
$$

where $k$ and $T$ are, respectively, the Boltzmann constant and the absolute temperature. In this expression, $U_{i 3}^{*}$ is the minimal potential energy of monomer molecule $i$ arising from the presence of its neighboring polymer when the mean distance between them is equal to Lennard-Jones' characteristic distance.

\section{Results and discussions}

The governing equations are solved numerically subject to the associated boundary conditions by a finite difference procedure. In numerical computations, the axial step, $d z$, and the radial step, $d r$, are selected to be first order and second order, respectively, in precision. According to the stability criterion suggested by Crank [20], under this constraint, $\left(D_{\max } / u_{z}\right)\left(d z / d r^{2}\right)<0.5$, where $D_{\max }$ is the maximal diffusivity, both the selected axial step and the radial step are decreased gradually until the solution converges. The physical properties of the materials used are summarized in Table 1 . The free volume fractions of monomers at various temperatures are estimated by referring to the thermal expansion data of $\mathrm{C}_{7} \mathrm{H}_{16}$ and $\mathrm{C}_{13} \mathrm{H}_{28}$ measured by Doolittle [23]. For amorphous PMMA, the glass transition temperature, $T_{\mathrm{g}}$, is $378 \mathrm{~K}$. According to Williams et al. [24], the free volume fraction of PMMA below its glass transition temperature can be estimated by $f_{3}=0.025$. The Flory-Huggins parameter for MMA, $\chi_{1}$, is assumed the value 0.45 [25]; also, $\chi_{2}=z_{2} \chi_{1}$ from Appendix A. The experimental conditions are summarized in Table 2. The interactive parameters $U_{11}^{*} / k$ and $U_{22}^{*} / k$ are estimated to be -450 and $-3000 \mathrm{~K}$, respectively, from the experimental refractive index data.

The applicability of the result derived in this study is examined by fitting the available experimental data in the literature. Figs. 3-6 illustrate the radial distribution of the refractive index under various conditions. Both the experimental data gathered from the literature and the results predicted by the present model are presented. For

Table 1

\begin{tabular}{|c|c|c|c|c|c|}
\hline & & MMA & BzMA & PMMA & PBzMA \\
\hline$d_{\mathrm{i}}\left(\mathrm{g} / \mathrm{cm}^{3}\right)$ & & 0.936 & 1.040 & 1.200 & 1.179 \\
\hline$m_{i}(\mathrm{~g} / \mathrm{mol})$ & & 100.12 & 176.22 & - & - \\
\hline$n_{\mathrm{Di}}$ & & - & - & 1.490 & 1.568 \\
\hline \multirow[t]{2}{*}{$f_{i}$} & $80 \mathrm{C}$ & 0.303 & 0.249 & 0.025 & - \\
\hline & $90 \mathrm{C}$ & 0.313 & 0.257 & 0.025 & - \\
\hline
\end{tabular}

Physical properties of MMA, BzMA, PMMA, and PbzMA [25,29]
Table 2

Experimental conditions for the data shown in Figs. 3-6

\begin{tabular}{llllll}
\hline & & I [14] & II [15] & III [15] & IV [15] \\
\hline \multirow{2}{*}{ Inner layer } & PMMA (\%) & 58 & 57.5 & 57.5 & 58 \\
& BzMA (\%) & 28 & 17 & 20 & 28 \\
& MMA (\%) & 14 & 25.5 & 22.5 & 14 \\
Outer layer & PMMA (\%) & 60 & 55.5 & 55.5 & 60 \\
& BzMA (\%) & 0 & 0 & 0 & 0 \\
& MMA (\%) & 40 & 44.5 & 44.5 & 40 \\
$R_{i}(\mathrm{~mm})$ & & 0.25 & 0.417 & 0.417 & 0.417 \\
$R_{\mathrm{o}}(\mathrm{mm})$ & & 0.5 & 0.833 & 0.833 & 0.833 \\
$u(\mathrm{~cm} / \mathrm{s})$ & & 4.244 & 4.244 & 4.244 & 4.244 \\
$L(\mathrm{~cm})$ & & 45 & 45 & 45 & 45 \\
\hline
\end{tabular}

comparison, the values predicted by other theoretical results are also shown. Figs. 3 and 4 reveal that the performance of the present model is satisfactory. In particular, the performance of the model proposed by Liu et al. [17] can be improved considerably by applying the present model. Note that the spatial distribution of the refractive index near the inner layer-outer layer interface is not as smooth as that at other position. This behavior may arise from the difference in the concentrations of polymers in the inner and the outer layers at the inlet. The critical free volume fractions and the estimated dilute diffusivities associated with Figs. 3-6 are summarized in Table 3. This table reveals that within the range of the operation conditions considered, the variation of the critical free volume fraction is more insensitive to the operation conditions than that of the dilute diffusivity of monomer. This is because the critical free volume fraction depends largely on the structure and the concentration of polymer chains. However, we are still able to see that the critical free volume fraction needed for the co-operative motion of polymer segments is reduced to a certain extent for the rotation energy of polymer segments increases as temperature rises. The dilute diffusivities of MMA and BzMA are found to increase

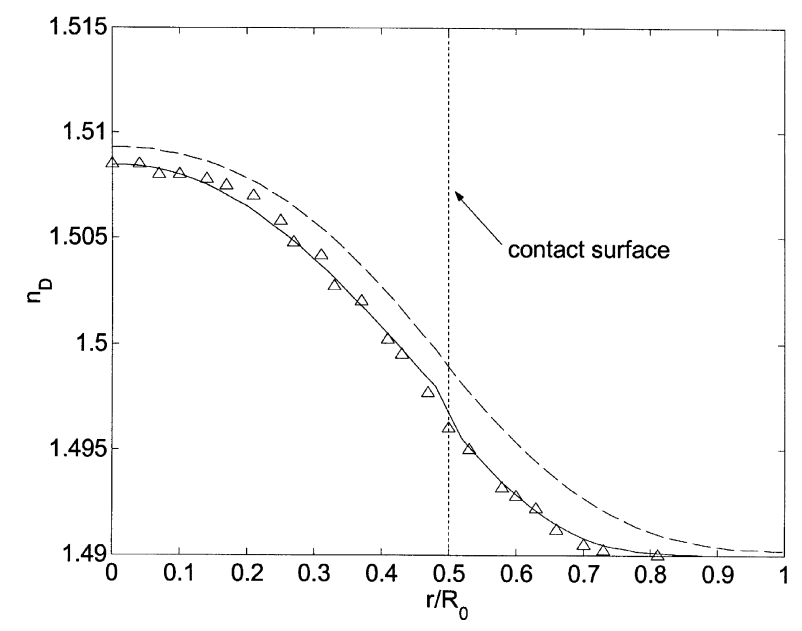

Fig. 3. Spatial variations of $n_{\mathrm{D}}$ at $80^{\circ} \mathrm{C}$. The experimental conditions are given in column I of Table 2. Discrete symbols, Ho et al. [14], dashed curve, Liu et al. [17], solid curve, present model. 


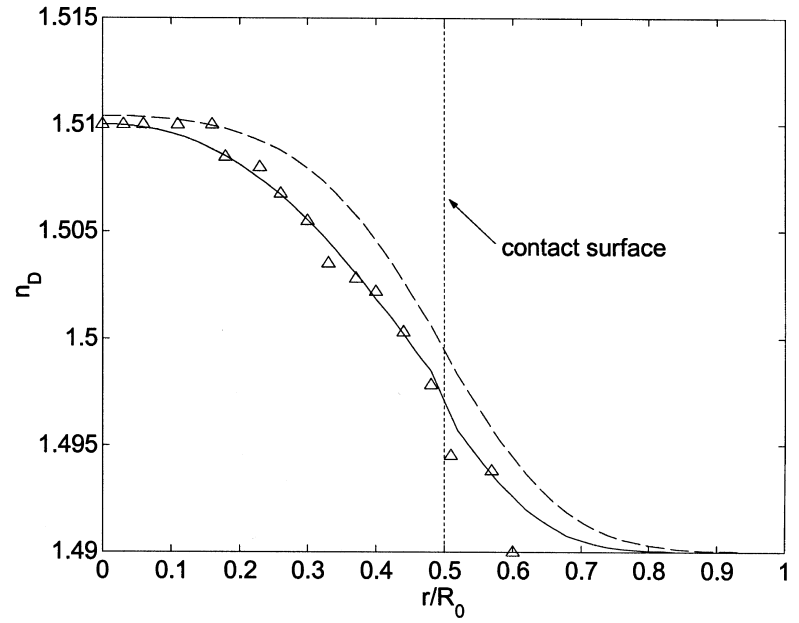

Fig. 4. Spatial variation of $n_{\mathrm{D}}$ at $90{ }^{\circ} \mathrm{C}$. The experimental conditions are given in column IV of Table 2. Discrete symbols, Chen et al. [15], dashed curve, Liu et al. [17], solid curve, present model.

with the increase in the temperature, and decrease with the increase in the critical free volume fraction. The latter is very sensitive.

Fig. 7 shows the radial distribution of the concentration of each component at the outlet of the diffusion zone for the case of Fig. 4. According to Fig. 7, the diffusion depth of BzMA is only about $20 \%$ of the radius of the optical fiber, which is responsible for the sharp decrease in the refractive index near the center of the fiber. The spatial variations of the diffusivities of MMA and BzMA are illustrated in Fig. 8. This figure indicates that for both components, the diffusivity in the outer layer is larger than that in the inner layer. This is mainly due to the fact that the free volume contribution of MMA is greater than that of BzMA, and the content of MMA in the outer layer is more abundant than that in the inner layer. Because polymer is distributed uniformly

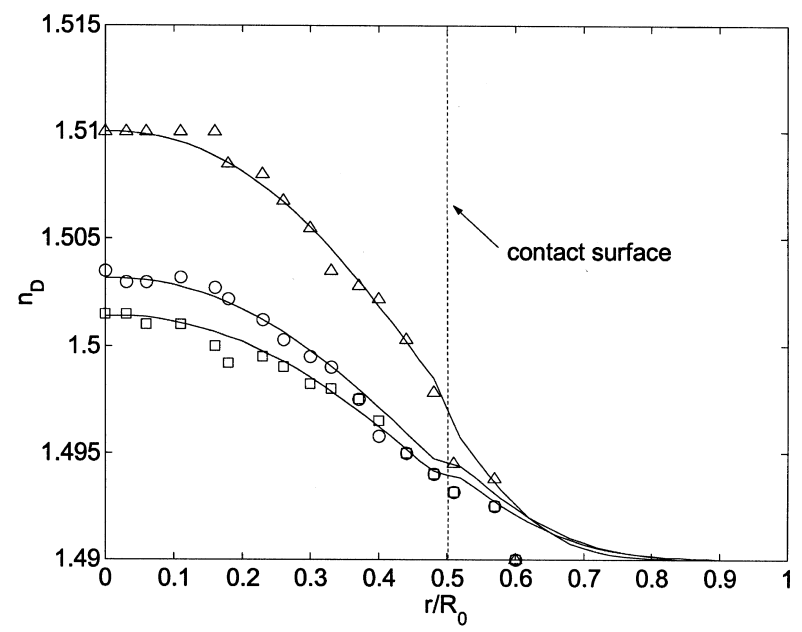

Fig. 5. Spatial variations of $n_{\mathrm{D}}$ at $90{ }^{\circ} \mathrm{C}$ for various inlet concentrations. Discrete symbols, Chen et al. [15], solid curve, present model. Experimental conditions for $\square, \bigcirc$, and $\Delta$, are given in columns II, III, and IV of Table 2, respectively.

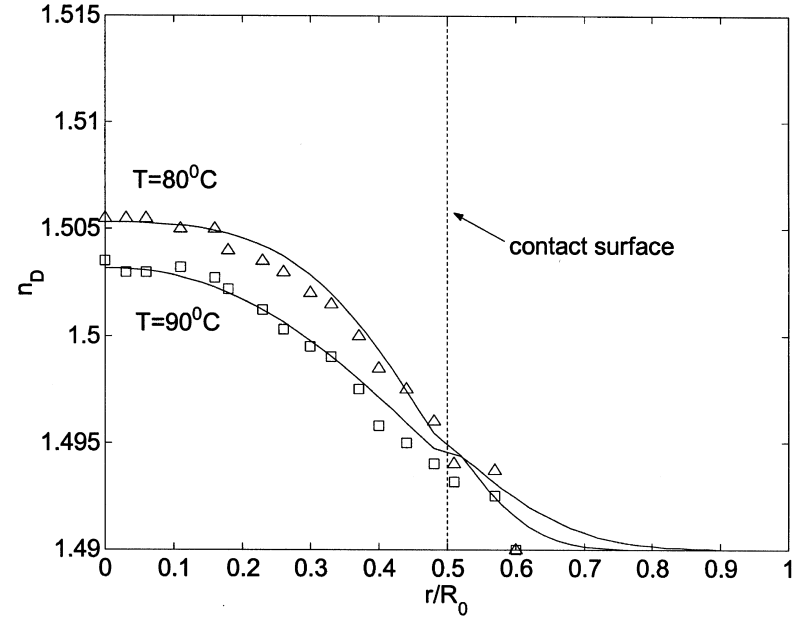

Fig. 6. Spatial variations of $n_{\mathrm{D}}$ at different temperatures. Discrete symbols, Chen et al. [15], solid curves, present model. Experimental conditions for $\Delta$ and $\square$ are given in columns III of Table 2 .

in both inner and outer layers, the spatial variations in the diffusivities of monomers observed in Fig. 8 indicate that the effect of the presence of one monomer on the diffusivity of the other monomer is very important. Fig. 8 and Table 3 suggest that the diffusivity of MMA in PMMA is about 10 times that of BzMA on the cross surface of fiber. This can be explained by that the molecular weight of BzMA is larger than MMA, and the attraction between BzMA and polymer chains is stronger than that between MMA and polymer chains.

In summary, a mathematical model is proposed for the description of the performance of a closed co-extrusion process for preparing gradient index plastic optical fiber. Based on a modified free volume theory, the present model is capable of taking the concentration-dependent diffusivity of monomer in a polymer solution into account, and we show that this effect can be significant. For instance, under typical conditions, the percentage deviation in the refractive index arising from the assumption of constant diffusivity can be on the order of $10.3 \%$. It should be pointed out that the present model does not limited to the parabolic-type refractive index distribution considered; it is also applicable to other types of refractive index distribution patterns.

Table 3

Critical free volume fractions and estimated dilute diffusivities of MMA and BzMA under various conditions

\begin{tabular}{llll}
\hline Experimental conditions & $f_{\mathrm{c}}$ & $D_{0,1}\left(\mathrm{~cm}^{2} / \mathrm{s}\right)$ & $D_{0,2}\left(\mathrm{~cm}^{2} / \mathrm{s}\right)$ \\
\hline I $\left(80^{\circ} \mathrm{C}\right)$ & 0.28 & $1.50 \times 10^{-9}$ & $1.52 \times 10^{-10}$ \\
II $\left(90^{\circ} \mathrm{C}\right)$ & 0.12 & $9.50 \times 10^{-7}$ & $1.01 \times 10^{-7}$ \\
III $\left(80^{\circ} \mathrm{C}\right)$ & 0.25 & $4.99 \times 10^{-9}$ & $5.03 \times 10^{-10}$ \\
III $\left(90^{\circ} \mathrm{C}\right)$ & 0.125 & $7.77 \times 10^{-7}$ & $8.29 \times 10^{-8}$ \\
IV $\left(90^{\circ} \mathrm{C}\right)$ & 0.19 & $5.77 \times 10^{-8}$ & $6.16 \times 10^{-9}$ \\
\hline
\end{tabular}




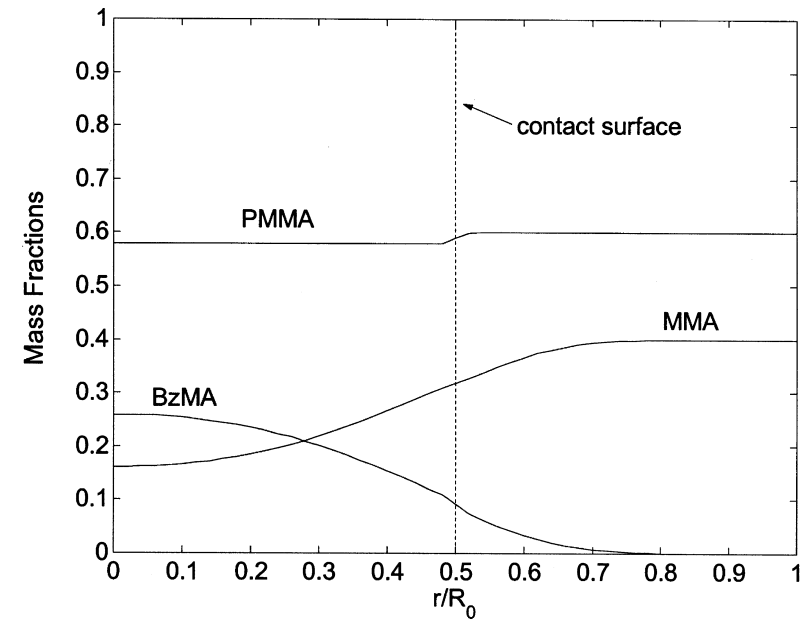

Fig. 7. Spatial variations of the mass fractions of MMA, BzMA, and PMMA at the outlet of diffusion zone. The experimental conditions are the same as that in Fig. 4.

\section{Acknowledgements}

This work is supported by the National Science Council of the Republic of China.

\section{Appendix A}

According to Fujita et al. [22], if the volume fraction of polymer is fixed, the diffusivity of monomer in a polymer solution can be estimated by

$D_{i}=D_{0, i} \exp \left(-\frac{f_{\mathrm{c}}}{f_{3}}+\frac{f_{\mathrm{c}}}{f}\right)\left(\frac{\partial \ln a_{i}}{\partial \ln \phi_{i}}\right)_{T, P, \phi_{3}}$

The last term on the right-hand side of this equation represents the non-ideality of monomer $i$, which can be evaluated by the procedure below. The total change of

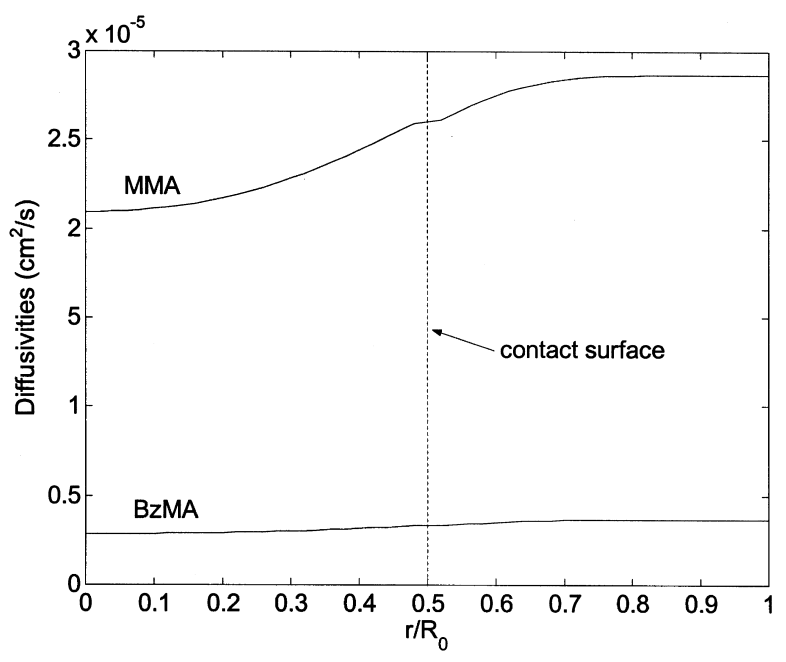

Fig. 8. Spatial variations of the diffusivities of MMA and BzMA at the outlet of diffusion zone. The experimental conditions are the same as that in Fig. 4. entropy for the formation of a (monomer $1+$ monomer $2+$ polymer 3 ) polymer solution, $\Delta S$, can be expressed as [26]

$$
\begin{array}{r}
\frac{\Delta S}{k}=\sum_{i=1}^{3}\left\{N_{i} \ln \left[\frac{\sum_{j=1}^{3}\left(N_{j} v_{j} f_{j}\right)}{N_{i} v_{i} f_{i}}\right]\right\} \\
=\sum_{i=1}^{3}\left\{N_{i} \ln \left[\frac{\sum_{j=1}^{3}\left(N_{j} z_{j} f_{j}\right)}{N_{i} z_{i} f_{i}}\right]\right\}
\end{array}
$$

where $N_{i}$ is the number of molecules of component $i$, and $v_{i}$ is the average volume occupied by each molecule of component $i$. The volume fraction of component $i$ can be expressed approximately by $\phi_{i}=N_{i} z_{i} / \sum_{j=1}^{3} N_{j} z_{j}$. Based on van Laar expression, the total change of enthalpy, $\Delta H$, can be expressed as [27]

$$
\begin{aligned}
\Delta H & =Z \Delta \omega_{23} N_{3} z_{3} \phi_{2}+Z \Delta \omega_{12} N_{1} \phi_{2} \\
& =Z \Delta \omega_{12} N_{2} z_{2} \phi_{3}+Z \Delta \omega_{12} N_{2} z_{2} \phi_{1} \\
& =Z \Delta \omega_{12} N_{2} z_{2}\left(1-\phi_{2}\right)=k T \chi_{2} N_{2}\left(1-\phi_{2}\right)
\end{aligned}
$$

where $\Delta \omega_{12}=\omega_{12}-\left(\omega_{11}+\omega_{22}\right) / 2=\Delta \omega_{23}, \Delta \omega_{13}=0$, and $\chi_{i}=Z \Delta \omega_{12} z_{i} / k T$ with $i=1,2$. In these expressions, $\omega_{i j}$ is the interactive energy for $(i-j)$ molecule (monomer or polymer unit) pair, and $Z$ denotes the coordinate number. The total change of free energy $\Delta F$ is $\Delta H-T \Delta S$, and the activity of component $i, a_{i}$, can be expressed by $k T \ln \times$ $a_{i}=\left(\partial \Delta F / \partial N_{i}\right)_{T, P, N_{j \neq i}}$. For a fixed volume fraction of polymer, we have

$$
\begin{aligned}
& \left(\frac{\partial \ln a_{1}}{\partial \ln \phi_{1}}\right)_{T, P, \phi_{3}}=1-\left(1-1 / z_{2}\right) \phi_{1}-2 \chi_{1} \phi_{2} \\
& \left(\frac{\partial \ln a_{2}}{\partial \ln \phi_{2}}\right)_{T, P, \phi_{3}}=1-\left(1-z_{2}\right) \phi_{2}-2 \chi_{2} \phi_{2}\left(1-\phi_{2}\right)
\end{aligned}
$$

Substituting these expressions into Eq. (A1) yields Eqs. (7a) and $(7 b)$ in the text.

If both the concentrations of MMA and BzMA are low, the main factors that are significant to the diffusivity of monomer $i$ are the root-mean-square velocity, $v_{\mathrm{rms}, i}$, and the activation energy, $\Delta E_{\mathrm{a}, i}$, of monomer $i$; the former is inversely proportional to the square root of the molecular weight of monomer $i$. The activation energy of monomer $i$ is equivalent to the interaction energy between the molecule of monomer $i$ and its neighboring polymer. The latter can be estimated from the free volume fraction of polymer solution, $f_{3}$. Employing Lennard-Jones 6-12 potential model [28], we have

$\Delta E_{\mathrm{a}, i}=U_{i 3}^{*}\left[\left(1-f_{3}\right)^{4}-2\left(1-f_{3}\right)^{2}\right]$ 
Therefore,

$$
\begin{aligned}
D_{0, i} & \propto v_{\mathrm{rms}, i} \exp \left(-\frac{\Delta E_{\mathrm{a}, i}}{k T}\right) \\
& =m_{i}^{-1 / 2} \exp \left\{-\frac{U_{i 3}^{*}\left[\left(1-f_{3}\right)^{4}-2\left(1-f_{3}\right)^{2}\right]}{k T}\right\}
\end{aligned}
$$

Eq. (8) can be recovered from this expression.

\section{References}

[1] Marchand EW. Gradient index optics. New York: Academic Press; 1978.

[2] Luneburg RK. Mathematical theory of optics. Berkeley: University of California Press; 1964.

[3] Morgan SP. J Appl Phys 1958;29:1358.

[4] Houde-Walter SN, Moore DT. Appl Opt 1985;24:4326.

[5] Koike Y, Ishigure T, Horibe A, Nihei E. Proceedings of Second International Conference on Plastic Optical Fibers and Application. The Hauge, June 28-29. Geneva: The European Institute for Communications and Networks; 1993.

[6] Koike Y, Sumi Y, Ohtsuka Y. Appl Opt 1986;25:3356.

[7] Koike Y, Kanemitsu A, Shioda Y, Nihei E, Ohtsuka Y. Appl Opt 1994;33:3394.

[8] Mishina Y, Uotsu Y, Oda M. Jpn. Patent. 1-189602, 1989.

[9] Mishina Y, Uotsu Y, Oda M. Jpn. Patent. 2-16505, 1990.

[10] Mishina Y, Uotsu Y, Oda M. Jpn. Patent. 2-33104, 1990.
[11] Tsai CC, Liu TJ, Chang YH, Tseng TW. Chem Engng Sci 1997;52: 221.

[12] Perry GA, Witcher CE. U.S. Patent, 5, 235, 660, 1993.

[13] Toyoda N, Mishina Y, Murata R, Oda M, Ishimaru T. U.S. Patent, 5, 390, 274, 1995.

[14] Ho BC, Chen JH, Chen WC, Chang YH, Yang SY, Chen JJ, Tseng TW. Polym J 1995;27:310.

[15] Chen WC, Chen JH, Yang SY, Cherng JY, Chang YH, Ho BC. J Appl Polym Sci 1996;60:1379.

[16] Van Krevelen DW. Properties of polymers. New York: Elsevier; 1976.

[17] Liu BT, Chen WC, Hsu JP. Polymer 1999;40:1451.

[18] Liu BT, Hsieh MY, Chen WC, Hsu JP. Polym J 1999;31:233.

[19] Chen WC, Chang Y, Hsu JP. J Phys Chem B 1999;103:7584.

[20] Crank J. The mathematics of diffusion. New York: Oxford University Press; 1992.

[21] Crank J, Park GS. Diffusion in polymers. New York: Academic Press; 1968.

[22] Fujita H, Kishimoto A, Matsumoto K. Trans Faraday Soc 1960;56: 424.

[23] Doolittle AKJ. Appl Phys 1951;22:1471.

[24] Williams ML, Landel RF, Ferry JD. J Am Chem Soc 1955;77:3701.

[25] Brandrup J, Immergut EH. Polymer handbook. New York: Wiley; 1966.

[26] Hildebrand JH. J Chem Phys 1947;15:225.

[27] Flory PJ. Principles of polymer chemistry. New York: Cornell University Press; 1967.

[28] Hsu JP, Lin SH. J Chem Phys 2003;118:172.

[29] Dean JA. Lange's handbook of chemistry. New York: McGraw-Hill; 1985. 